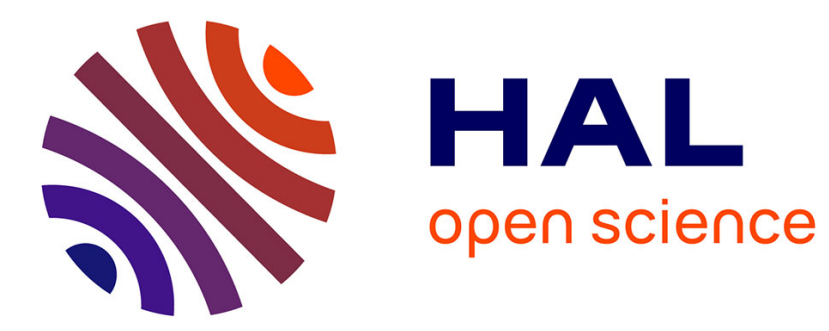

\title{
Rough paths: an introduction using classical analysis
}

Antoine Lejay

\section{To cite this version:}

Antoine Lejay. Rough paths: an introduction using classical analysis. Numerical Nalaysus abd Applied Mathematics (ICNAAM), Sep 2007, Corfou, Greece. pp.339-342. inria-00200339

\section{HAL Id: inria-00200339 \\ https://hal.inria.fr/inria-00200339}

Submitted on 20 Dec 2007

HAL is a multi-disciplinary open access archive for the deposit and dissemination of scientific research documents, whether they are published or not. The documents may come from teaching and research institutions in France or abroad, or from public or private research centers.
L'archive ouverte pluridisciplinaire HAL, est destinée au dépôt et à la diffusion de documents scientifiques de niveau recherche, publiés ou non, émanant des établissements d'enseignement et de recherche français ou étrangers, des laboratoires publics ou privés. 


\section{Rough paths: an introduction using classical analysis}

Antoine Lejay $^{1}$ — Projet TOSCA (INRIA / Institut Élie Cartan, Nancy, France)

Abstract: In this article, we give a simple presentation of the theory of rough paths, that relies only on simple considerations on integrals along smooth paths and convergence of families of Hölder continuous functions.

Keywords: Rough path; controlled differential equations; Heisenberg group; Brownian motion; stochastic differential equations; Young integrals

AMS Classification: 60H10; 93C10

Published in Numerical Analysis and Applied Mathematics . Simos, T.E., Psihoyios, H., Tsitouras, Ch. (eds.), AIP Conference Proceedings (American Institute of Physics), 936, 339-342, 2007

${ }^{1}$ Current address: Projet TOSCA

Institut Élie Cartan, UMR 7502, Nancy-Université, CNRS, INRIA,

Campus scientifique

BP 239

54506 Vandœuvre-lès-Nancy CEDEX, France

E-mail: Antoine.Lejay@iecn.u-nancy.fr 
A. Lejay / Rough paths: an introduction using classical analysis

\section{Introduction}

The theory of rough paths is a way to give a positive answer to the following questions:

(Q1) Given a smooth enough differentiable form $f$ on $\mathbb{R}^{d}(d \geq 1)$, is it possible to define a map $\mathfrak{K}$ on the space $\mathcal{C}^{\alpha}\left([0, T] ; \mathbb{R}^{d}\right)$ of Hölder continuous functions from $[0, T]$ to $\mathbb{R}^{d}$ whose restriction to the space $\mathcal{C}_{\mathrm{p}}^{1}\left([0, T] ; \mathbb{R}^{d}\right)$ of piecewise functions of class $\mathcal{C}^{1}$ is

$$
\mathfrak{K}(x):=\left(t \mapsto \int_{0}^{t} f\left(x_{s}\right) \mathrm{d} x_{s}\right)
$$

and such that $\mathfrak{K}$ is continuous.

(Q2) Is it possible to extend continuously to $\mathcal{C}^{\alpha}\left([0, T] ; \mathbb{R}^{m}\right)$ the map $\mathfrak{I}(x)=y$ where $y$ is solution to the controlled differential equation

$$
y_{t}=y_{0}+\int_{0}^{t} g\left(y_{s}\right) \mathrm{d} x_{s},
$$

where $g$ is a smooth enough function from $\mathbb{R}^{m}$ to the set of linear functions $\mathcal{L}\left(\mathbb{R}^{d} ; \mathbb{R}^{m}\right)$ and $x \in \mathcal{C}_{\mathrm{p}}^{1}([0, T] ; \mathbb{R})$.

It is well known that it is a priori not possible to do so if $\alpha \leq 1 / 2$ and $d \geq 2$. For example, if $x$ is a Brownian motion trajectory, defining $\int_{0}^{t} f\left(x_{s}\right) \mathrm{d} x_{s}$ requires special constructions such as Itô or Stratonovich integrals, and there are known counter-examples to the continuity properties (See for example [1]). If $d=1$, then $\mathfrak{K}$ is easily constructed using a primitive of $f$, and $\mathfrak{I}$ can be defined with an approach in the style of the one of Doss and Sussman [2, 3].

However, T. Lyons and his co-authors [4, 5] (see also [6]) have shown how it to complete this program by replacing the increments $x_{s, t}=x_{t}-x_{s}$ in a Riemann-sum like definition of $\mathfrak{K}$ as

$$
\mathfrak{K}(x)_{t}=\lim _{n \rightarrow \infty} \sum_{k=0}^{2^{n}-1} f\left(x_{t k 2^{-n}}\right) x_{t k 2^{-n}, t(k+1) 2^{-n}}
$$

by some elements $\mathbf{x}_{s, t}$ living in a tensor space $\mathbb{R} \oplus \mathbb{R}^{d} \oplus\left(\mathbb{R}^{d}\right)^{\otimes 2} \oplus \cdots \oplus\left(\mathbb{R}^{d}\right)^{\lfloor 1 / \alpha\rfloor}$ and how to defined $\mathfrak{K}$ as the limit of

$$
\mathfrak{K}(\mathbf{x})_{t}=\lim _{n \rightarrow \infty} \sum_{k=0}^{2^{n}-1} \sum_{i=1}^{\lfloor 1 / \alpha\rfloor} \nabla^{i-1} f\left(x_{t k 2^{-n}}\right) \pi_{\left(\mathbb{R}^{d}\right) \otimes i}\left(\mathbf{x}_{\left.t k 2^{-n}, t(k+1) 2^{-n}\right)}\right),
$$

where $\pi_{\left(\mathbb{R}^{d}\right)^{\otimes i}}$ is the projection on $\left(\mathbb{R}^{d}\right)^{\otimes i}$ for $i=1,2, \ldots$ The map $\mathfrak{I}$ can then be defined using a fixed point theorem. Since then, several authors 
have presented alternative constructions and points of view [7, 8, 9]. Note also that there also exists some infinite dimensional versions of this theory [10, 11]. The goal of this paper is to justify from simple considerations on integrals why the construction of T. Lyons is indeed natural. For this, we mix ideas borrowed from P. Friz, N. Victoir, A. de La Pradelle, D. Feyel, ... The article [12] presents a survey of these ideas.

\section{$2 \quad$ Young integrals}

The $\alpha$-Hölder norm is denoted by $\|\cdot\|_{\alpha}$. The space $\mathcal{C}^{\alpha}\left([0, T] ; \mathbb{R}^{d}\right)$ of Hölder continuous function is continuously embedded in $\mathcal{C}^{\beta}\left([0, T] ; \mathbb{R}^{d}\right)$ for any $\beta \leq \alpha$. We denote by $\mathcal{H}^{\alpha, \beta}\left(\mathbb{R}^{d}\right)$ the space $\mathcal{C}^{\alpha}\left([0, T] ; \mathbb{R}^{d}\right)$ equipped with the Hölder norm $\|\cdot\|_{\beta}$ for $\beta \leq \alpha$. This distinction is important, since:

- The space $\mathcal{C}^{\infty}\left([0, T] ; \mathbb{R}^{d}\right)$ is dense in $\mathcal{H}^{\alpha, \beta}\left(\mathbb{R}^{d}\right)$ for any $\beta<\alpha \leq 1$ but not in $\mathcal{H}^{\alpha, \alpha}\left(\mathbb{R}^{d}\right)=\mathcal{C}^{\alpha}\left([0, T] ; \mathbb{R}^{d}\right)$.

- There exists sequences of functions $\left(x^{n}\right)_{n \in \mathbb{N}}$ with $x^{n}$ in $\mathcal{C}^{\alpha}\left([0, T] ; \mathbb{R}^{d}\right)$ and such that $x^{n}$ converges in $\|\cdot\|_{\beta}$ with $\beta<\alpha$ to some $x \in \mathcal{C}^{\alpha}\left([0, T] ; \mathbb{R}^{d}\right)$, but that the convergence does not hold in $\|\cdot\|_{\alpha}$, as we will see it later.

We now split $\cup_{0<\beta \leq \alpha \leq 1} \mathcal{H}^{\alpha, \beta}\left(\mathbb{R}^{d}\right)$ into several zones, as shown in Figure 1. The map $\mathfrak{K}$ will then be constructed on $\mathcal{H}^{\alpha, \beta}$, which means that the existence of $\mathfrak{K}(x)$ depends on the Hölder regularity of $x$, but its continuity depends on the Hölder norm that ensures the convergence of a family of $\alpha$-Hölder paths. The answer to (Q1) and (Q2) depends on the part of the triangle $(\alpha, \beta)$ belongs to. Theorem 1 below answers to (Q1) and (Q2) for $\alpha \geq \beta>1 / 2$, i.e., in Zone I.

In 1936, L.C. Young [13] showed how to construct a bilinear map $(x, y) \mapsto$ $\left(t \mapsto \int_{0}^{t} y_{s} \mathrm{~d} x_{s}\right)$ from $\mathcal{H}^{\alpha, \alpha}\left(\mathbb{R}^{d}\right) \times \mathcal{H}^{\beta, \beta}\left(\mathbb{R}^{d}\right)$ to $\mathcal{H}^{\alpha, \alpha}(\mathbb{R})$ which is continuous, provided that $\alpha+\beta>1$. When applied with $y_{t}=f\left(x_{t}\right)$ for a function $f$ of

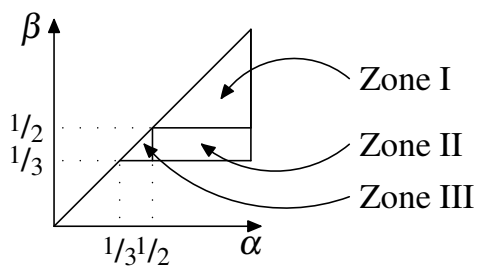

Figure 1: The spaces of Hölder continuous functions with Hölder exponent $\alpha$ and Hölder norm $\beta$. 
class $\mathcal{C}^{\gamma}$, then necessarily, one needs $\alpha(\gamma+1)>1$ and then, one can apply this result only for $\alpha>1 / 2$ at best. However, when $\alpha>1 / 2$, the maps $\mathfrak{K}$ and $\mathfrak{I}$ from the introduction may be defined using Young integrals. It has to be noted that the regularity of $\mathfrak{K}(x)$ and $\mathfrak{I}(x)$ is the one of $x$. The next theorem (see [4, 6]) summarizes these results.

Theorem 1. If $f \in \mathcal{C}^{\gamma}$ and $g \in \mathcal{C}^{1}$ with $\nabla g \in \mathcal{C}^{1+\gamma}$ are smooth enough, then $\mathfrak{I}$ and $\mathfrak{K}$ are defined using Young integrals and are continuous from $\mathcal{H}^{\alpha, \beta}$ to $\mathcal{H}^{\alpha, \beta}$ for $\alpha \geq \beta$ and $\beta(1+\gamma)>1$ (and then $\alpha \geq \beta>1 / 2$ ).

\section{The Green-Riemann formula}

We consider now a path $x$ in $\mathcal{C}^{\alpha}\left([0, T] ; \mathbb{R}^{2}\right)$ (here, we restrict without loss of generality to the dimension $d=2$ ). and the set of piecewise linear approximations $x^{n}$ of $x$ along the family of dyadic partitions $\left\{\left\{k T / 2^{n}\right\}_{k=0, \ldots, 2^{n}}\right\}_{n \geq 0}$ of $[0, T]$. Now, let $\phi$ be an arbitrary function in $\mathcal{C}^{1}([0, T] ; \mathbb{R})$, and consider the approximation $\widehat{x}^{n}$ of $x$ construct by the following way: On $\left[k T / 2^{n}, k T / 2^{n}+\right.$ $\left.2^{n-1}\right], \widehat{x}^{n}$ moves at constant speed along a loop starting at $x_{k T / 2^{n}}$ with radius $\phi\left((k+1) T / 2^{n}\right)-\phi\left(k T / 2^{n}\right)$. On $\left[k T / 2^{n}+2^{n-1},(k+1) T / 2^{n}\right], \widehat{x}^{n}$ moves at constant speed along the segment joining $x_{k T / 2^{n}}$ to $x_{(k+1) T / 2^{n}}$.

Now, let $f$ be a smooth differential form. Using the Chasles relation and the Green-Riemann formula,

$$
\begin{array}{r}
\int_{0}^{T} f\left(\widehat{x}_{s}^{n}\right) \mathrm{d} \widehat{x}_{s}^{n}=\int_{0}^{T} f\left(x^{n}\right) \mathrm{d} x_{s}^{n}+\sum_{k=0}^{2^{n}-1} \iint_{\operatorname{Area}}\left(\widehat{x}_{\mid\left[k T / 2^{n}, k T / 2^{n}+2^{n+1}\right]}^{n}[f, f](z) \mathrm{d} z\right. \\
\approx \int_{0}^{T} f\left(x^{n}\right) \mathrm{d} x_{s}^{n}+\sum_{k=0}^{2^{n}-1}[f, f]\left(x_{k T / 2^{n}}\right)\left(\phi\left((k+1) T / 2^{n}\right)-\phi\left((k+1) T / 2^{n}\right)\right) \\
\underset{n \rightarrow \infty}{\longrightarrow} \int_{0}^{T} f(x) \mathrm{d} x_{s}+\int_{0}^{T}[f, f]\left(x_{s}\right) \mathrm{d} \phi_{s},
\end{array}
$$

with $[f, f]=\partial_{x_{2}} f_{1}-\partial_{x_{1}} f_{2}$. Thus, the limit of $\mathfrak{K}\left(\widehat{x}^{n}\right)$ is different from the limit of $\mathfrak{K}\left(x^{n}\right)$, which is $\mathfrak{K}(x)$. The fact is that $x^{n}$ converges to $x$ in $\mathcal{H}^{\alpha, \beta}$ for $\beta<\alpha$, while $\widehat{x}^{n}$ converges to $x$ in $\mathcal{H}^{\beta, \gamma}$ for $\gamma<1 / 2$, unless $\phi$ is constant.

The idea to extend $\mathfrak{K}$ to paths with values in $\mathbb{R}^{3}$ is now justified by the fact that the sequences $\left(x^{n}\right)_{n \in \mathbb{N}}$ and $\left(\widehat{x}^{n}\right)_{n \in \mathbb{N}}$ have the same limit but $\left(\mathfrak{K}\left(x^{n}\right)\right)_{n \in \mathbb{N}}$ and $\left(\mathfrak{K}\left(\widehat{x}^{n}\right)\right)_{n \in \mathbb{N}}$ have different limits that are characterized by the presence of the term $\int_{0}^{0}[f, f]\left(x_{s}\right) \mathrm{d} \phi_{s}$. We now turn this to rigorous results. 


\section{Path with values in the Heisenberg group}

Let $(\mathcal{G}, \boxplus)$ be the Lie group made of elements of $\mathbb{R}^{3}$ with the operator $(x, y, z) \boxplus\left(x^{\prime}, y^{\prime}, z^{\prime}\right)=\left(x+x^{\prime}, y+y^{\prime}, \frac{1}{2}\left(x y^{\prime}-y x^{\prime}\right)\right)$. This is the Heisenberg group. This group $\mathcal{G}$ may be equipped with a sub-Riemannian distance $d(a, b)$ which is such that

$$
\begin{aligned}
& c \max \left\{\left|b^{1}-a^{1}\right|,\left|b^{2}-a^{2}\right|, \sqrt{\left|b^{3}-a^{3}\right|}\right. \leq d(a, b) \\
& \leq C \max \left\{\left|b^{1}-a^{1}\right|,\left|b^{2}-a^{2}\right|, \sqrt{\left|b^{3}-a^{3}\right|}\right\}
\end{aligned}
$$

for some constants $c, C>0$ and all $a, b \in \mathcal{G}$ (see [9, 14]). For $a=(x, y, z) \in \mathcal{G}$, $-a=(-x,-y,-z)$ is its inverse. The distance $d$ is such that $d(a, b)=$ $d(0,(-a) \boxplus b)$.

Let us consider paths $\mathbf{x}$ with values in $\mathcal{G}$. We $\operatorname{define}$ set $\mathcal{C}^{\alpha}([0, T] ; \mathcal{G})$ of $\alpha$-Hölder continuous paths with values in $\mathcal{G}$ as the set of paths $\mathbf{x}$ such that

$$
\|\mathbf{x}\|_{\mathcal{G}, \alpha}:=\sup _{0 \leq s<t \leq T} \frac{d\left(\mathbf{x}_{s}, \mathbf{x}_{t}\right)}{(t-s)^{\alpha}}<+\infty
$$

We now denote by $\mathcal{H}^{\alpha, \beta}(\mathcal{G})$ the space of $\mathcal{G}$-valued $\alpha$-Hölder continuous function equipped with the norm $\|\cdot\|_{\mathcal{G}, \beta}$.

Let us denote by $\mathfrak{A}$ the map from $\mathcal{C}^{\alpha}\left([0, T] ; \mathbb{R}^{2}\right)$ when $\alpha>1 / 2$ to $\mathcal{C}([0, T] ; \mathcal{G})$ defined by

$$
\mathfrak{A}(x):=\left(t \mapsto\left(x_{t}^{1}, x_{t}^{2}, \frac{1}{2} \int_{0}^{t}\left(x_{s}^{1}-x_{0}^{1}\right) \mathrm{d} x_{s}^{2}-\frac{1}{2} \int_{0}^{t}\left(x_{s}^{2}-x_{0}^{2}\right) \mathrm{d} x_{s}^{1}\right)\right),
$$

where the integrals $\int x^{i} \mathrm{~d} x^{j}, i, j \in\{1,2\}$ are defined as Young integrals. For any $t \geq 0$, the quantity $\mathfrak{A}(x)_{t}$ is the algebraic area enclosed between $x_{\mid[0, t]}$ and the segment that joins $x_{0}$ to $x_{t}$.

The next proposition asserts that, when $\alpha>1 / 2, \mathfrak{A}$ defines a one-to-one map between $\mathcal{C}^{\alpha}\left([0, T] ; \mathbb{R}^{2}\right)$ and $\mathcal{C}^{\alpha}([0, T] ; \mathcal{G})$.

Proposition 1. For $\alpha>1 / 2$, $\mathfrak{A}$ maps continuously $\mathcal{H}^{\alpha, \alpha}\left(\mathbb{R}^{2}\right)$ to $\mathcal{H}^{\alpha, \alpha}(\mathcal{G})$. Conversely, if $\mathbf{x} \in \mathcal{C}^{\alpha}([0, T] ; \mathcal{G})$, then there exists $x \in \mathcal{C}^{\alpha}\left([0, T] ; \mathbb{R}^{2}\right)$ such that $\mathbf{x}=\mathfrak{A}(x)$.

The next theorem now explain how to extend $\mathfrak{K}$ to Zone II in Figure 1 .

Theorem 2. Let $x$ be a path in $\mathcal{C}^{\alpha}\left([0, T] ; \mathbb{R}^{2}\right)$ with $\alpha>1 / 2$ and $\phi$ in $\mathcal{C}^{\beta}([0, T] ; \mathbb{R})$ with $\beta>2 / 3$. Let $f=f_{1} \mathrm{~d} x_{1}+f_{2} \mathrm{~d} x_{2}$ be a differential form on $\mathbb{R}^{2}$ such that $f_{i} \in \mathcal{C}^{1}$ and $\nabla f_{i} \in \mathcal{C}^{\epsilon}$ with $\alpha \epsilon+\beta>1$, Then there exists a sequence $\left(\widehat{x}^{n}\right)_{n \in \mathbb{N}}$ in $\mathcal{C}_{\mathrm{p}}^{1}\left([0, T] ; \mathbb{R}^{2}\right)$ such that $\mathfrak{A}\left(x^{n}\right)$ converges to $\mathbf{y}=$ 
$\left(\mathbf{x}^{1}, \mathbf{x}^{2}, \mathbf{x}^{3}+\phi\right)$ in $\mathcal{H}^{\beta / 2, \gamma}(\mathcal{G})$ with $\mathbf{x}=\mathfrak{A}(x)$ and $\gamma<\beta / 2$. In addition, $\mathfrak{K}\left(x^{n}\right)$ converges in $\mathcal{H}^{\alpha \wedge \beta, \delta}(\mathbb{R})(\delta<\alpha \wedge \beta)$ to

$$
\mathfrak{K}(\mathbf{y}):=\left(t \mapsto \int_{0}^{t} f\left(x_{s}\right) \mathrm{d} x_{s}+\int_{0}^{t}[f, f]\left(x_{s}\right) \mathrm{d} \phi_{s}\right) .
$$

On the other hand, if $\left(x^{n}\right)_{n \in \mathbb{N}}$ is the sequence of linear interpolations of $x$ on the dyadics, then $\mathfrak{K}\left(x^{n}\right)$ converges in $\mathcal{H}^{\alpha, \delta}(\mathbb{R})$ to $\mathfrak{K}(\mathbf{x}):=\mathfrak{K}(x) \in \mathcal{C}^{\alpha}([0, T] ; \mathbb{R})$ for any $\delta<\alpha$.

The sequence $\left(\widehat{x}^{n}\right)_{n \in \mathbb{N}}$ of this theorem is indeed constructed the same way we constructed the sequence $\left(\widehat{x}^{n}\right)_{n \in \mathbb{N}}$ in the previous Section. We may also join the points $\left\{x_{k T / 2^{n}}\right\}_{k=0, \ldots, 2^{n}}$ using sub-Riemannian geodesics [9, 12].

The path $\mathbf{y}$ above is a path in $\mathcal{C}^{\beta / 2}([0, T] ; \mathcal{G})$, while the path $\mathbf{x}$ belongs to $\mathcal{C}^{\alpha}([0, T] ; \mathcal{G})$. The difference between $\mathbf{y}$ and $\mathbf{x}$ lies in the function $\phi$. However, both $\mathbf{x}$ and $\mathbf{y}$ projects on $\mathbb{R}^{2}$ onto the same path $x$ (we then say that $\mathbf{x}$ and $\mathbf{y}$ lies above the path $x$ ). We may then identify $\mathbf{x}$ and $\mathbf{y}$ with two different sequences $\left(x^{n}\right)_{n \in \mathbb{N}}$ (the family of piecewise liner interpolations of $x$ ) and $\left(\widehat{x}^{n}\right)_{n \in \mathbb{N}}$, the latter one being defined using the "extra" function $\phi$.

There is no need to restrict to a path which is regular.

Theorem 3. Let $\mathbf{x}$ be a path in $\mathcal{C}^{\alpha}([0, T] ; \mathcal{G})$ with $\alpha>1 / 3$. Then there exists a sequence of paths $\left(x^{n}\right)_{n \in \mathbb{N}}$ with $x^{n} \in \mathcal{C}_{\mathrm{p}}^{1}\left([0, T] ; \mathbb{R}^{2}\right)$ such that $\mathbf{x}^{n}:=\mathfrak{A}\left(x^{n}\right)$ converges to $x$ in $\mathcal{H}^{\alpha, \beta}(\mathcal{G})$ with $\beta<\alpha$. For a differential form $f=f_{1} \mathrm{~d} x_{1}+$ $f_{2} \mathrm{~d} x_{2}$ with $f_{i} \in \mathcal{C}^{1}$ and $\nabla f_{i} \in \mathcal{C}^{\gamma}$ with $\alpha(1+\gamma)>1$, $\mathfrak{K}\left(x^{n}\right)$ converges in $\mathcal{H}^{\alpha, \beta}(\mathcal{G}), \beta<\alpha$, to some path in $\mathcal{C}^{\alpha}([0, T] ; \mathbb{R})$ which we denote by $\mathfrak{K}(\mathbf{x})$.

In addition, the map $\mathfrak{K}$ is continuous from $\mathcal{H}^{\alpha, \beta}(\mathcal{G})$ to $\mathcal{H}^{\alpha, \beta}(\mathcal{G})$ with $\alpha>$ $1 / 3, \beta<\alpha$.

We have to note that for $1 / 3<\alpha<1 / 2$, this theorem defines $\mathfrak{K}(\mathbf{x})$ on a space of functions that take their values in $\mathbb{R}^{3}$, since for $\mathbf{x} \in \mathcal{C}^{\alpha}([0, T] ; \mathcal{G})$ and $\phi \in \mathcal{C}^{2 \alpha}([0, T] ; \mathbb{R}), \mathbf{y}:=\left(\mathbf{x}^{1}, \mathbf{x}^{2}, \mathbf{x}^{3}+\phi\right)$ belongs to $\mathcal{C}^{\alpha}([0, T] ; \mathcal{G})$ and $\mathfrak{K}(\mathbf{y})=\mathfrak{K}(\mathbf{x})+\int_{0}^{\cdot}[f, f]\left(x_{s}\right) \mathrm{d} \phi_{s}$ with $x=\left(\mathbf{x}^{1}, \mathbf{x}^{2}\right)$.

Theorem 3 together with Theorem 2 means that to be properly extended to $\alpha$-Hölder continuous $\mathbb{R}^{2}$-valued functions with $1 / 3<\alpha \leq 1 / 2, \mathfrak{K}$ needs to be defined on $\mathcal{C}^{\alpha}([0, T] ; \mathcal{G})$, which means that we use paths that are genuine $\mathbb{R}^{3}$-valued paths. The question is how to define paths in $\mathcal{C}^{\alpha}([0, T] ; \mathcal{G})$ and then to extend $\mathfrak{K}$ to Zone III in Figure 1]? There is non-canonical way to "lift" a path $x \in \mathcal{C}^{\alpha}\left([0, T] ; \mathbb{R}^{2}\right)$ to a path $\mathbf{x} \in \mathcal{C}^{\alpha}([0, T] ; \mathcal{G})$ when $\alpha \leq 1 / 2$. However, the next theorem, borrowed from [15], asserts it is always possible to consider such paths.

Theorem 4. Let $x$ be a path in $\mathcal{C}^{\alpha}\left([0, T] ; \mathbb{R}^{2}\right)$ with $1 / 3<\alpha \leq 1 / 2$. Then there exists a path $\mathbf{x}$ in $\mathcal{C}^{\alpha}([0, T] ; \mathcal{G})$ that lies above $x$. 
However, in some specific cases, such as trajectories of stochastic processes, one can be more precise. For example, for the trajectories $B(\omega)$ of a 2-dimensional Brownian motion, one can consider the family of piecewise linear approximations $\left(B^{n}(\omega)\right)_{n \in \mathbb{N}}$ along dyadic partitions of $[0, T]$ and show that $\mathfrak{A}\left(B^{n}(\omega)\right)$ converges in $\mathcal{C}^{\alpha}([0, T] ; \mathcal{G})$ for any $\alpha<1 / 2$ to some path $\mathbf{B}(\omega)$. Using the continuity results and the Wong-Zakai theorem [1], this proves that, with this family of approximations, $\mathfrak{K}(\mathbf{B})$ is equal to the Stratonovich integral $\int_{0}^{\cdot} f\left(B_{s}\right) \circ \mathrm{d} B_{s}$. Other choices of approximations may lead to different integral, and we then recover the counter-examples to the Wong-Zakai theorem due to McShane [1]. Itô integrals may also be constructed using this theory, but one has to deal with the brackets of the Brownian motion, and it is impossible to find a sequence $\left(\widehat{B}^{n}\right)_{n \in \mathbb{N}}$ of piecewise smooth paths such that $\mathfrak{K}\left(\widehat{B}^{n}\right)$ converges to the Itô integral $\int_{0}^{*} f\left(B_{s}\right) \mathrm{d} B_{s}$. On these subject, see [4, 5, 6, 6,12$]$.

If one want to deal with $\alpha<1 / 3$, then one needs to use iterated integrals up to order $\lfloor 1 / \alpha\rfloor$. However, the geometrical interpretation of such a construction has not been done yet.

The advantage of the theory of rough paths in order to define an integral of a differential form along an irregular path $x$ or to solve a differential equation controlled by an irregular path $x$ is that one has only to focus on the construction of $\mathfrak{A}(x)$, which means that one has to construct the integrals of $x$ along itself. From this construction, one can construct $\mathfrak{K}$ for any differential form $f$ smooth enough, and also $\mathfrak{I}$ for a smooth enough function $g$ using a fixed point theorem. For this, one may use a family of piecewise linear approximations of $x$, and for stochastic processes, some tools that are similar to the one in the proof of the Kolmogorov-Censtov theorem. This approach has already been used for a wide variety of stochastic processes: see the book [16] for example.

\section{References}

[1] N. Ikeda, and S. Watanabe, Stochastic Differential Equations and Diffusion Processes, North-Holland, 1989, 2 edn. 2, 7

[2] H. Doss, Ann. Inst. H. Poincaré Sect. B (N.S.) 13, 99-125 (1977). 2

[3] H. Sussmann, Ann. Probability 6, 19-41 (1978). 2

[4] T. Lyons, Rev. Mat. Iberoamericana 14, 215-310 (1998). 2, 4, 7

[5] T. Lyons, and Z. Qian, System Control and Rough Paths, Oxford Mathematical Monographs, Oxford University Press, 2002. 2, 7 
A. Lejay / Rough paths: an introduction using classical analysis

[6] A. Lejay, "An introduction to rough paths," in Séminaire de probabilités, XXXVII, Springer-Verlag, 2003, vol. 1832 of Lecture Notes in Mathematics, pp. 1-59. 2, 4, 7

[7] M. Gubinelli, J. Funct. Anal. 216, 86-140 (2004). 3

[8] D. Feyel, and A. de La Pradelle, Electron. J. Probab. 11, 860-892 (2006). 3

[9] P. Friz, and N. Victoir, Probab. Theory Related Fields 136, 395-416 (2006a). 3, 5, 6]

[10] M. Ledoux, T. Lyons, and Z. Qian, Ann. Probab. 30, 546-578 (2002). 3

[11] R. Léandre, Chen-Souriau calculus for rough loop (2003), preprint. 3

[12] A. Lejay, Yet another introduction to rough paths (2006), URL http: //hal.inria.fr/inria-00107460, preprint. 3, 6, (7)

[13] L. Young, Acta Math. 67, 251-282 (1936). 3

[14] R. Montgomery, A tour of subriemannian geometries, their geodesics and applications, vol. 91 of Mathematical Surveys and Monographs, American Mathematical Society, 2002. 5

[15] T. Lyons, and N. Victoir, An extension theorem to rough paths (2005), preprint. 6

[16] P. Friz, and N. Victoir, Multidimensional Stochastic Processes as Rough Paths. Theory and Applications, Cambridge University Press, 2006b, in preparation. 7 